\title{
Phacomorphic Glaucoma: Evolving Management Strategies
}

\author{
Shibal Bhartiya, Mahesh Kumar HM, Mohit Jain \\ Dr RP Center for Ophthalmic Sciences, All India Institute of Medical Sciences, New Delhi, India
}

\section{INTRODUCTION}

Phacomorphic glaucoma is defined as secondary angle-closure glaucoma due to an increase in lens thickness and is characterized by a sudden rise in IOP which can compromise the function of the optic nerve and may lead to irreversible visual loss if not treated in time. This increase in lens thickness can be due to an advanced cataract, a rapidly intumescent lens, or a traumatic cataract, eventually leading to pupillary block and angle closure.

Phacomorphic glaucoma is peculiar to developing countries like India, (constitutes 3.91\% of all cataract operations done) where the incidence of cataract far exceeds the total number of surgeries. ${ }^{1-5}$ In developing countries, due to an unequal distribution of eye care facilities and economic constraints, many patients with age related cataracts are not able to get cataract surgery in time and present with phacomorphic glaucoma. In the European races, there is a gradual shrinkage of lens with development of cataract and thereby a progressive deepening of the anterior chamber occurs. Although no formal epidemiological data exists comparing the geographical and racial predilections, cataracts in Indians seem to show sudden hydration and intumescence rather commonly.

\section{MECHANISM}

The primary mechanism responsible for angle closure is a thickened and anteriorly displaced lens (due to zonular laxity with age and sometimes compounded by pseudoexfoliation), although pupillary block may also play a role in disease pathophysiology (Figs 1 and 2).

Therefore the rise in IOP is due to the pupillary block caused by a combination of changes in the size of the lens and the forward displacement of the lens iris diaphragm, resulting in angle closure. Generally, phacomorphic glaucoma is observed in older patients with senile cataracts, but it can occur in younger patients after a traumatic cataract or a rapidly developing intumescent cataract. If not managed in time, permanent synechial closure of the angle can occur with persistently elevated IOP even after the removal of the cataractous lens.

\section{CLINICAL FEATURES}

\section{Symptoms and Signs}

Phacomorphic glaucoma is recognized by the complaints of pain and redness in an eye which has had a progressive painless diminution of vision. On examination there is injection of conjunctival and episcleral vessels, corneal edema, shallow anterior chamber, an intumescent cataractous lens. An asymmetric central shallowing of the anterior chamber in the presence of a unilateral mature intumescent cataract and elevated IOP alerts the examiner to its possibility (Fig. 1). Occasionally, there may be a precipitating incident such as pharmacological pupil dilation.

Angra et $\mathrm{al}^{3}$ reported that phacomorphic glaucoma is a disease of old age, the mean age at presentation being 64 years with none occurring below 50 years, and a preponderance between $50-60$ years.

The age range in both types of LIG, phacolytic and phacomorphic, as reported by Pradhan et al was 40 to 80 years, with 145 of the 413 (35\%) cases occurring in patients aged under 60 years of age. The female to male ratio was $1.7: 1 .^{1}$

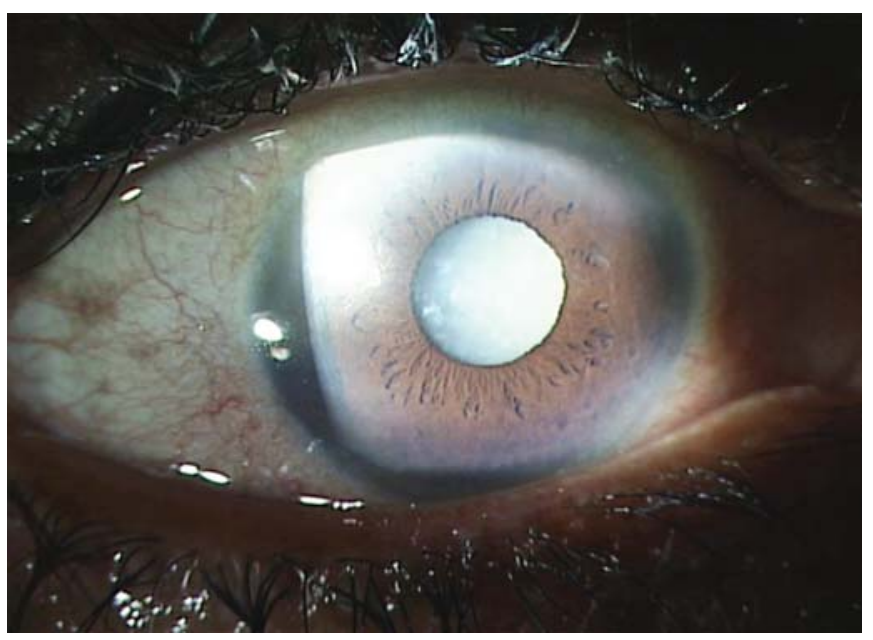

Fig. 1: Intumescent lens with shallow AC and circumciliary congestion 


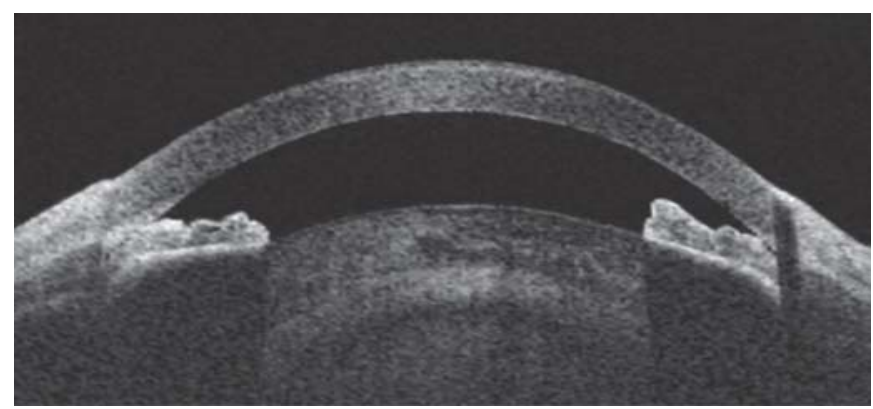

Fig. 2: ASOCT picture showing shallowing of the anterior chamber due to shift in the lens iris diaphragm because of intumescent lens

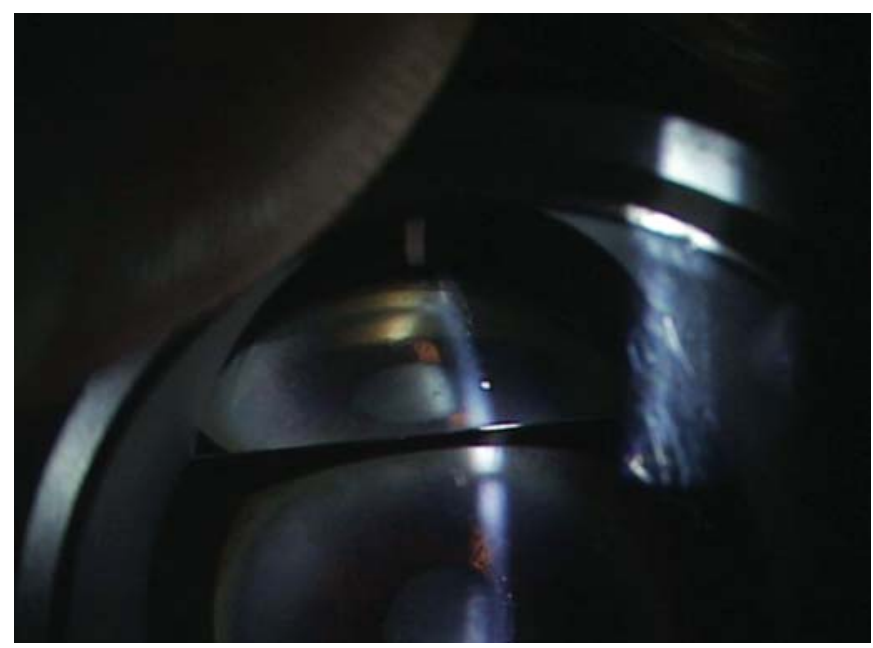

Fig. 3: Goniophotograph of a case of phacomorphic glaucoma

In another study, female preponderance was found to be almost three times more. They attributed this to the lesser attention received by old women for medical treatment in rural India and also the fact that anatomically, females have a shallower anterior chamber depth thus making them more prone for angle closure. The incidence and rise of IOP was related to the maturity of cataract. The duration of attack is related to the type of cataract: lesser with intumescent cataract, whereas no relation could be found with the height of raised intraocular pressure. $^{4}$

Pain of more than 10 days duration in the affected eye was the chief presentation in $71 \%$ patients. Patients came from within $50 \mathrm{~km}$ of the hospital, and $62.4 \%$ had to travel more than $100 \mathrm{~km}$ to reach the hospital. The major reasons for late presentation were "no escort" in 34.6\% and "lack of money" in 31.0\%cases. Other reasons included "lack of time" in 7.0\%, "no desire for surgery" in $6.5 \%$, "uncertainty about where to go" in $6.1 \%$, "feeling of adequate vision" in 5.3\%, "no mature cataract" in 4.6\%, "fear in $4.4 \%$ and "uncontrolled systemic diseases" (diabetes mellitus and hypertension) in $0.5 \%$ patients. $^{1}$

\section{CLINICAL EVALUATION}

A thorough general physical examination and ophthalmic evaluation including corneal clarity and thickness, pupillary reaction, AC inflammation and gonioscopy is mandatory (Fig. 3). Specular biomicroscopy, including endothelial counts and morphology, as well as B scan ultrasonography for posterior segment evaluation must be performed whenever possible, in addition to routine investigations for cataract surgery.

An endothelial cell loss of $14.8 \%$ has been reported by Angra et al after the glaucomatous attack. The authors compared the cell counts with that of the fellow eye since the preglaucoma endothelial count was not available, assuming that the endothelial cell counts in the two eyes are comparable. ${ }^{3}$

The optic disk also shows changes which are significantly related to the duration of attack glaucoma. Jain et al reported that in attacks lasting for more than 3 weeks, nearly all the eyes developed pallor, cupping or atrophy of the disk. Up to 10 days of attack, a large majority of optic disks (76.2\%) retained good color. ${ }^{5}$

\section{TREATMENT}

Medical therapy is started immediately to lower IOP, control inflammation and salvage any potential vision. If possible, a laser peripheral iridotomy (LPI) should be performed to treat the pupillary block. A prophylactic LPI in the fellow eye is a prudent recourse. Once IOP is controlled medically and the eye is quiet, cataract surgery can be performed. If the patient presents after 72 hours and indentation gonioscopy reveals synechial closure, a combined surgery is usually required. ${ }^{3-5}$

It has been reported that these eyes tend to withstand raised intraocular pressure for a longer period than expected. At least $54 \%$ of the eyes with less than 2 days duration of attack recovered $6 / 12$ or better vision, whereas only $32 \%$ of the eyes recovered this visual acuity if the duration of attack lasted 3 to 5 days as reported by Jain et al. ${ }^{5}$ Thus, as the duration of attack increased there was a progressive decline in the recovery of visual acuity and beyond three weeks only light preception or hand movements could be recovered.

\section{MEDICAL MANAGEMENT}

Control of the raised IOP is the first line management of phacomorphic glaucoma, followed by control of the intraocular inflammation and corneal edema. The role of medical therapy, thus, is extremely important in the management of phacomorphic glaucoma.

Preoperative control of IOP using systemic hyperosmolar agents (mannitol and glycerol), oral and topical acetazolamide, and topical antiglaucoma medication including beta blockers and alpha agonists is recommended. Topical steroids may be prescribed to lower inflammation and hypertonic saline for corneal edema. 
Angra et al found that intraocular pressure in $37.5 \%$ eyes could not be controlled medically. These eyes were found to have extensive peripheral anterior synechiae (PAS), and a longer duration of attack. Visual status also could be improved after medical management, out of the 16 cases which presented with faulty projection of light, 9 cases reversed to have an accurate light projection after bringing down the IOP. The sudden increase in IOP can lead to a decrease in perfusion pressure and optic nerve ischemia with conduction defects leading to an inaccurate projection of light which may be reversed if treated at an early stage with IOP lowering. ${ }^{3}$

\section{ROLE OF PERIPHERAL IRIDECTOMY AND IRIDOPLASTY}

Most cases of phacomorphic glaucoma reach the ophthalmologist at a late stage when peripheral anterior synechiae have developed and the closure of angle is either partly or totally structural rather than only functional.

Tomey et al reported that of the 10 patients, who were treated for phacomorphic glaucoma, by first undergoing neodymium:YAG (Nd:YAG) laser iridotomy, the acute angleclosure glaucoma attack could be reversed or prevented by the iridotomy, before the subsequent cataract extraction. ${ }^{6}$

Yip et al reported that IOP was successfully controlled in 17 eyes (80.75\%) of the 21 patients in whom argon laser iridoplasty was performed as the initial step in the management of phacomorphic glaucoma. They reported a statistically significant decrease at 2 and 24 hours postoperatively, concluding that ALPI is a safe and efficacious measure for the initial management of phacomorphic glaucoma, simultaneously obviating the need to operate in highly inflamed eyes in an emergency setting and achieving satisfactory mid-term visual outcome. $^{7}$

Tham et al also reported that immediate ALPI, replacing systemic antiglaucoma medications, appeared to be safe and effective as first-line treatment of acute phacomorphic angleclosure. $^{8}$

$13.95 \%$ of patients evaluated by Jain et al showed evidence of phacomorphic glaucoma in the opposite eye, $50 \%$ of whom had been operated previously and 50\% had gone into absolute stage.

They however, do not recommend prophylactic iridectomy on the fellow eyes of these patients because the phacomorphic attack seems to occur almost 10 years later than the acute congestive glaucoma indicating that it is the swollen cataract per se that is responsible for this attack rather than a narrow angle alone. They postulated that the surgical procedure itself may accelerate the formation of a hydrated cataract and found that iridectomy did not prevent an acute attack in 3 cases. Thus it appears that in these cases there is an acute angle closure by forward push of the iris root rather than a physiologic pupil block and iris bombe as seen in acute closed angle glaucoma. Peripheral iridectomy in such a situation may be insufficient to prevent an acute attack of phacomorphic glaucoma. Instead these eyes should be kept under observation for development of cataract and cataract extraction should be done before development of the intumescent stage. ${ }^{5}$ In view of the fact that laser iridotomy is an easy outpatient procedure, we would recommend that fellow eyes be treated atleast to prevent the element of pupillary block since these patients may not come for regular follow-up.

\section{SURGICAL CONSIDERATIONS}

The usual surgical treatment recommended for such cases is to control the intraocular pressure first by medical therapy and to extract the lens later by performing a second operation (Figs 4 A to $6 \mathrm{~B}$ ). In case the IOP is not controlled, a second stage filtration surgery maybe performed. If there is a synechial closure of more than 270 degrees, a combined cataract and filtration surgery may be the procedure of choice.

Preoperative rise of intraocular pressure, accuracy of light projection and final visual recovery are significantly related to the duration of glaucoma. A good functional recovery may be obtained if the attack is of a short duration. In a study by Jain et al, in cases with attack duration more than 20 days only a hand movement or perception of light could be recovered. ${ }^{5}$

Table 1 provides an overview of the surgical procedures available, and the pro and cons of each.

Table 1: Surgical procedures: Brief overview

\begin{tabular}{|c|c|c|}
\hline Surgical procedure & Pros & Cons \\
\hline Phacoemulsification & $\begin{array}{l}\text { No conjunctival scarring, better cortical clean-up, } \\
\text { closed chamber procedure }\end{array}$ & $\begin{array}{l}\text { Clear cornea required, availability of instruments and } \\
\text { expertise maybe lacking in remote areas, corneal } \\
\text { endothelium already compromised }\end{array}$ \\
\hline ECCE/SICS & $\begin{array}{l}\text { No special instrumentation, } \\
\text { expertise required }\end{array}$ & $\begin{array}{l}\text { Conjunctival scarring may jeopardize future filtering } \\
\text { surgery }\end{array}$ \\
\hline Combined surgery & $\begin{array}{l}\text { Single stage procedure, } \\
\text { Faster rehabilitation }\end{array}$ & $\begin{array}{l}\text { Special expertise required, } \\
\text { Higher failure rates }\end{array}$ \\
\hline Cataract surgery with AGV & Experimental & $\begin{array}{l}\text { Special expertise required, Availability and expense of } \\
\text { AGV is a consideration }\end{array}$ \\
\hline
\end{tabular}



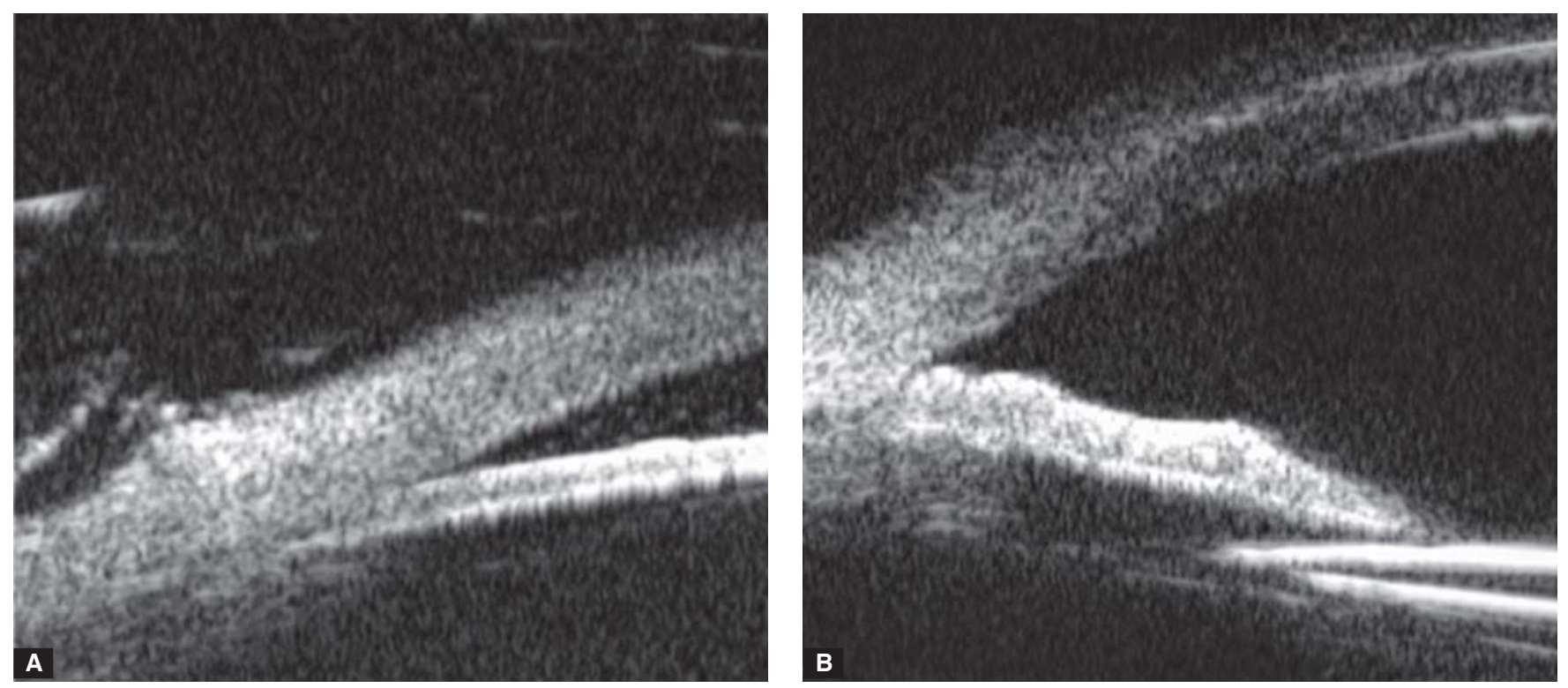

Figs 4A and B: Pre- and postoperative UBM picture of superior angle in phacomorphic glaucoma showing appositional closure and open angles following removal of intumescent lens
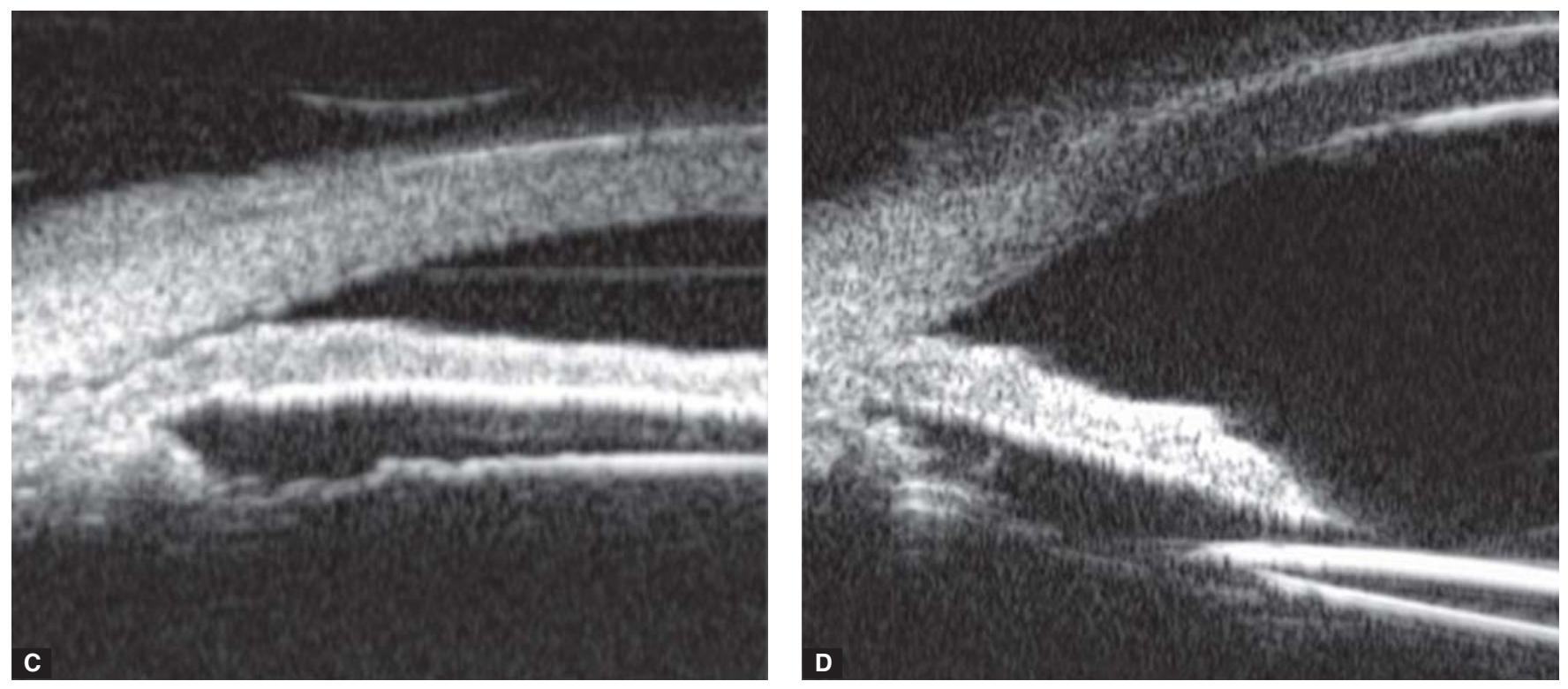

Figs 4C and D: Pre- and postoperative UBM picture of inferior angle in phacomorphic glaucoma showing appositional closure and open angles following removal of intumescent lens 


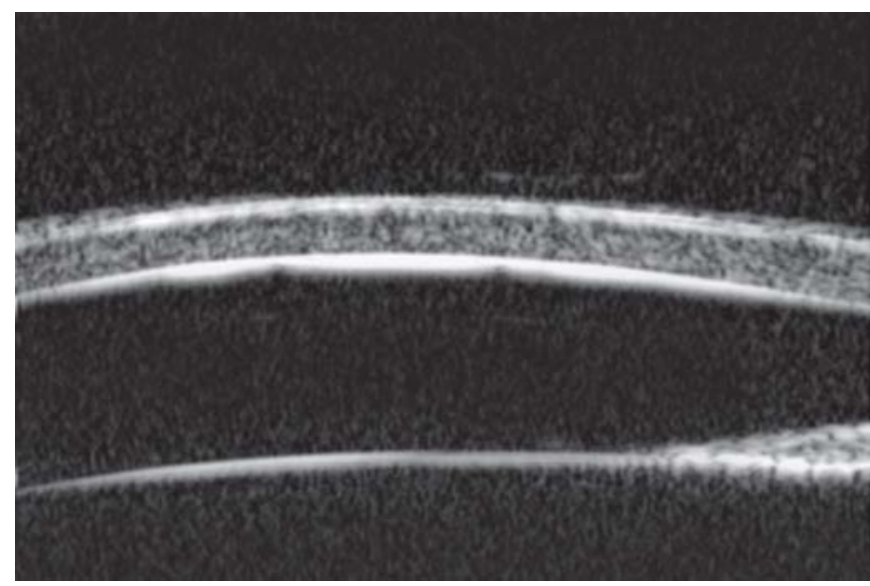

Fig. 5A: Preoperative anterior chamber as seen on UBM

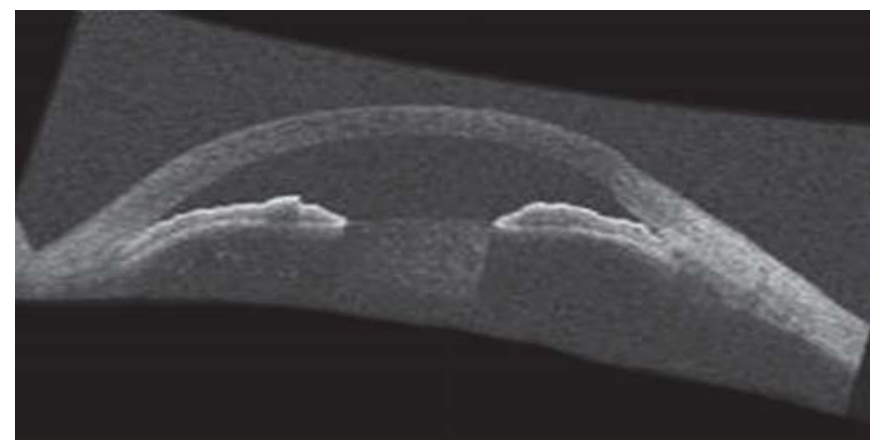

Fig. 6A: Preoperative SLOCT picture of phacomorphic glaucoma

\section{ECCE/SICS}

Extracapsular cataract extraction/Manual SICS with a sulcusfixated intraocular lens (IOL) can be performed but is associated with increased intraoperative and postoperative complications in this condition. ${ }^{5,9,10}$ Precautions that must be taken to optimize visual results are as follows:

Care must be taken to control inflammation and IOP preoperatively. Guarded ocular decompression is mandatory. A small pupil often requires the use of sphincterotomy, stretch pupilloplasty, iris hooks or pupillary dilators. Dye assisted envelop capsulotomy is preferred over can opener technique. Generous use of high viscosity viscoelastic to deepen the AC, and a viscodispersive like Viscoat to protect the corneal endothelium can improve visual outcome. The corneoscleral section must be adequate for the large nucleus. Meticulous cortical clean up is mandatory to minimize postoperative fibrinoid reaction, together with minimal handling of the iris. Use of heparin surface modified IOL (HSM PCIOL) placed in the bag is recommended.

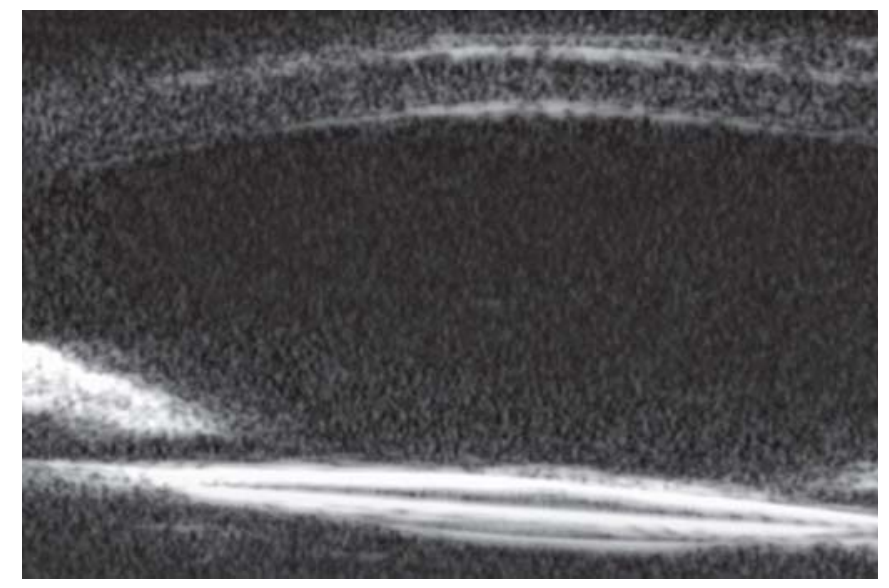

Fig. 5B: Postoperative UBM picture showing increase in AC depth following cataract surgery

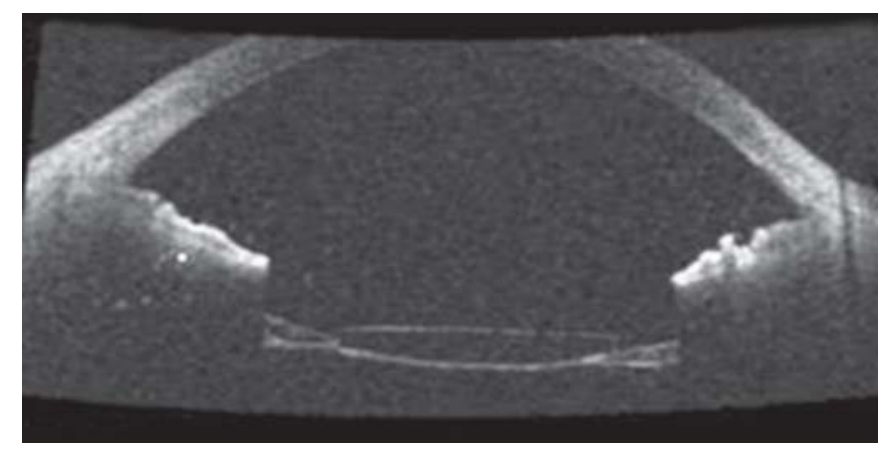

Fig. 6B: Postoperative SLOCT picture of phacomorphic glaucoma showing deepening of $A C$ and wide open angles

137 out of 229 (59.8\%) of the patients of phacomorphic glaucoma had a poor visual outcome. Pradhan et al reported that the distance from the hospital, duration of pain, and high IOP at presentation were all associated with poor visual outcome. ${ }^{1}$

Prajna et al reported that patients with age more than 60 years and in whom the glaucoma was present for more than 5 days had a significantly higher risk of poor visual outcome postoperatively. ${ }^{10}$

\section{PHACOEMULSIFICATION}

After the IOP has been lowered, clear corneal temporal phacoemulsification is preferred as it leaves the superior site free for a trabeculectomy if needed. In both ECCE and SICS, the resultant conjunctival scarring significantly reduces the success rate of subsequent filtering surgeries. ${ }^{9}$ The anterior chamber should be deepened by reducing the vitreous pressure by either a vitreous tap ${ }^{11}$, traditional 3 port pars plana vitrectomy or 23G/ $25 \mathrm{G}$ sutureless vitrectomy. ${ }^{12,13}$ 
Operating in a crowded eye which has a compromised endothelium and high IOP can lead to a significant risk of complications. Hence the authors recommend the technique of sutureless pars plana limited vitrectomy to lower IOP and deepen the anterior chamber prior to phacoemulsification surgery. ${ }^{13}$

The corneal endothelium should be protected using Viscoat and chilled BSS.

A small pupil is often encountered which requires the use of sphincterotomy, stretch pupilloplasty, iris hooks or pupillary dilators like Beehlers and Malyugin.

Absence of a fundal glow makes dye assisted capsulorhexis mandatory.

Rao $^{14}$ developed a technique that allows a safe, controlled capsulorhexis in the presence of the shallow anterior chamber, increased anterior capsule convexity, and high intralenticular pressure seen in eyes with phacomorphic glaucoma. The intumescent cataracts are decompressed by filling the anterior chamber with viscoelastic; a 30 gauge needle is then used to aspirate liquid cortex, which facilitates a controlled capsulorhexis. This allows safe phacoemulsification of the cataract and in-the-bag IOL implantation

Capsular tension ring should be kept handy for management of any zonular dehiscence.

Phacoemulsification should be done in the bag or in the iris plane, using low power, and flow rates, with an increased bottle height. Phacochop nucleotomy with burst mode, torsional and cold phacoemulsification all decrease postoperative inflammation and endothelial damage and are consequently preferred. ${ }^{15}$

Careful irrigation aspiration, preferably bimanual, is better in these cases. A hydrophobic, square edge foldable IOL (acrylic) is preferred. ${ }^{9}$

\section{COMBINED SURGERY}

Although cataract extraction and PCIOL implantation yields good visual results in phacomorphic glaucoma, a combined glaucoma and cataract surgery results in superior IOP control. Inadequate IOP control with maximal medical therapy (> 3 medications) and symptom duration $>3-7$ days is considered an indication for combined surgery.

Trabeculectomy with intraoperative Mitomycin C has been found to be more efficacious in lowering IOP in conjunction with phacoemulsification in comparison to its combination with ECCE. Both same site and two site phacotrabeculectomy have demonstrated similar visual results and IOP reduction. ${ }^{10,15-18}$

The type of conjunctival flap does not influence the final outcome. A fornix based flap has the advantage of shorter surgical time and faster visual rehabilitation; though it has an increased incidence of bleb leakage. ${ }^{17}$ If combined surgery is performed antifibrotic agents such as Mitomycin C should be used.

Since doing an ECCE/SICS disturbs the conjunctiva for future glaucoma surgery and doing a combined surgery in an inflamed eye is not associated with a favorable long-term IOP control, the best option would be to do a clear corneal Phacoemulsification with IOL (Figs 3 and 4 ) as this can lead to opening up of the angle and IOP control with / without medical therapy in majority of cases. If IOP is not controlled, subsequently a trabeculectomy with Mitomycin C can be done after a period of 3-6 months.

\section{AGV WITH ECCE}

Das et al reported that ECCE with HSM PCIOL and Ahmed glaucoma valve was an effective treatment modality for the management of phacomorphic glaucoma. AGV implantation and simultaneous cataract extraction with IOL implantation was performed through two separate incisions in patients diagnosed with phacomorphic glaucoma. The authors reported a steady control of IOP in all patients with minimum antiglaucoma medications. Superior preoperative IOP control and a shorter duration of the symptoms resulted in better postoperative vision. ${ }^{19}$

\section{PROBLEMS IN MANAGING PHACOMORPHIC GLAUCOMA}

Operating on phacomorphic glaucoma is a high risk situation as there is a significant risk of intraoperative and postoperative complications:

1. Decreased endothelial count

2. Corneal edema with decreased visibility

3. Anterior chamber inflammation

4. Decreased AC depth, less space for instrument maneuver

5. Positive lenticular pressure, increased risk of extension of capsulorhexis

6. Weak zonules

7. Iris prolapse

8. Small pupil

9. Risk of hemorrhage (esp. expulsive)

10. Hard cataract

11. Positive vitreous pressure

12. Increased chance of PC tear

13. Persistent IOP elevation requiring medical/surgical therapy

14. Increased risk of postoperative fibrinoid reaction

15. Poor visual outcome due to irreversible ONH damage.

\section{POSTOPERATIVE REGIMEN}

Meticulous postoperative care can improve the visual results even in phacomorphic glaucoma. Potent topical steroids (prednisolone acetate drops 1\% ) at a frequency of $1 / 2$ to 1 hourly, with topical antibiotic cover (broad spectrum fourth generation quinolone) to combat inflammation and prevent infection are imperative. Systemic corticosteroids may be added in patients with severe inflammation. 
IOP recordings and control with topical and systemic antiglaucoma therapy is extremely important, especially in case where a cataract extraction alone has been done. Care of the bleb is mandatory in combined extractions.

Proper refractive correction for both near and distance for visual rehabilitation must be provided. Visual fields and $\mathrm{ONH}$ evaluation must be carried out as soon as feasible

The patient must be advised surgery for the other eye, preferably the patient should not be sent home without surgery on the other eye as these patients often do not have access to health care. In case vitrectomy has been done, peripheral screening to rule out any retinal tear is mandatory.

In spite of successful surgery and meticulous postoperative care, the visual prognosis of this subgroup of patients often remains guarded. The causes for poor visual gain following surgery are enumerated in Table $2{ }^{1}$

Table 2: Cause of poor postoperative vision following surgery ${ }^{1}$

1. Optic atrophy

2. Uveitis

3. Corneal edema

4. Posterior capsular opacification

5. Amblyopia/unknown

6. Retinal/vitreous hemorrhage

7. Retinal detachment

8. Hyphema

9. Corneal opacity

10. Papillitis

\section{MEDICOLEGAL ASPECT}

Diagnosing phacomorphic glaucoma is as important as its subsequent management. It is important that the IOP be controlled medically, and the pupillary block removed. Cataract surgery is required in all cases of phacomorphic glaucoma, and the guarded visual prognosis must be explained to the patient in great detail. A special, written consent must be taken from the patient after explaining to him/her the risks and benefits of the procedure, as also the treatment options available. Also, as the posterior segment evaluation is not possible due to media haze, an ultrasound B-scan can preclude surprises during and after surgery.

al-Torbak et al reported a case with clinical findings typical of phacomorphic glaucoma. Preoperative ultrasonography revealed an intraocular tumor, magnetic resonance imaging and histopathological examination of the tumor after enucleation confirmed the diagosis. They concluded that clinical findings characteristic of phacomorphic glaucoma may be associated with choroidal melanoma. Ultrasonography or other imaging studies should be performed on eyes with phacomorphic glaucoma and opaque ocular media to allow timely detection of any mass lesion and to guide appropriate surgical management. ${ }^{20}$

\section{CONCLUSION}

Some patients in the poorest parts of the world with cataract wait for free eye camp surgery in the vicinity of their homes rather than visit a distant hospital for treatment for reasons both social and economic.

It is indeed a matter of grave consternation that more than sixty percent of the patients are symptomatic for more than 10 days before reaching a health care practitioner. Poor transportation and bad roads make travelling difficult, two thirds of the patients do not have access to an escort to the hospital and consequently do not reach the hospital on time. A similar percentage does not reach the health care practitioners due to economic constraints.

As is true for all other cases of glaucoma, the importance of long-term follow-up to assess IOP control and field loss cannot be over emphasized. Public health education programs to increase awareness and community support, upgradation of eye care delivery services in rural areas for early referral and treatment of cataract cases can help in primary prevention of this condition. Also improving the peripheral health care infrastructure for postoperative care as well as management of the fellow eye can prevent the incidence of visual loss, and the consequent economic burden on the community at large.

With the advent of superior methods of intraocular pressure control, and sophisticated instrumentation for cataract surgery, the visual prognosis in these patients has also become significantly better.

The concept of social justice and equitable distribution of health care with timely intervention on the part of the eye surgeon, will undoubtedly benefit this extremely neglected subgroup of patients of preventable blindness.

\section{REFERENCES}

1. Pradhan D, Hennig A, Kumar J, Foster A. Indian J Ophthalmol. A prospective study of 413 cases of lens-induced glaucoma in Nepal 2001;49:103-07.

2. McKibbin M, Gupta A, Atkins AD. J Cataract Refract Surg. Cataract extraction and intraocular lens implantation in eyes with phacomorphic or phacolytic glaucoma 1996;22:633-36.

3. Angra SK, Pradhan R, Garg SP. Indian J Ophthalmol. Cataract induced glaucoma: An insight into management 1991;39:97-101.

4. Verma BMD, Srivastava SK, Rekha. Proc. Ac. Ind. Ophthalmol Soc 1980;39:316.

5. Jain IS, Gupta A, Dogra MR, Gangwar DN, Dhir SP. Indian J Ophthalmol. Phacomorphic glaucoma: Management and visual prognosis 1983;31:648-53.

6. Tomey KF, al-Rajhi, AA Ophthalmology. Neodymium: YAG laser iridotomy in the initial management of phacomorphic glaucoma 1992;99:660-65. 
7. Yip PP, Leung WY, Hon CY, Ho CK. Ophthalmic Surg Lasers Imaging. Argon laser peripheral iridoplasty in the management of phacomorphic glaucoma 2005;36:286-91.

8. Tham CC, Lai JS, Poon AS, Chan JC, Lam SW, Chua JK, Lam DS. Eye. Immediate argon laser peripheral iridoplasty (ALPI) as initial treatment for acute phacomorphic angle-closure (phacomorphic glaucoma) before cataract extraction: A preliminary study 2005;19:778-83.

9. Tezel G ,Kolker AE ,Kass MA ,Wax MB. Ophthalmic Surg and lasers.Comparitve results of combined procedures for glaucoma and cataract:Extracapsular cataract extraction versus phcoemulsification and rigid versus foldable intraocular lenses 1997;28:539-50.

10. Prajna NV, Ramakrishnan R, Krishnadas R, Manoharan N Indian J Ophthalmol. Lens induced glaucomas: Visual results and risk factors for final visual acuity 1996;44:149-55.

11. Chang DF. J Cataract Refract Surg. Parsplana vitreous tap or phacoemulsification in the crowded eye 2001;27:1911-14.

12. Dada T, Kumar S, Gadia R, et al. J Cataract Refract Surg. Sutureless single-port transconjunctival parsplana limited vitrectomy combined with phacoemulsification for management of phacomorphic glaucoma 2007;33:951-54.

13. Chalam KV, Gupta SK, Agarwal S, Shah VA. Ophthalmic Surg and Lasers Imaging. Sutureless limited vitrectomy for positive vitreous pressure in cataract surgery 2005;36:518-22.

14. Rao SK, Padmanabhan P. J Cataract Refract Surg. Capsulorhexis in eyes with phacomorphic glaucoma 1998;24:882-84.

15. Burrato L. Phacoemulsification in glaucomatous eyes: Cataract surgery in complicated cases. Slack Incorp Pg-182.

16. Dada T, Muralidhar R, Sethi HS, BMC Ophthalmol. Insertion of foldable hydrophobic IOL through the trabeculectomy fistula in cases with microincision cataract surgery combined with trabeculectomy 2006;19(6):14.
17. Kozobolis VP, Siganos CS, Christadoulakis EV, Lazrov NP. J Cataract Refract Surg. Two site phacotrabeculectomy with intraoperative mitomycin C; Fornix versus limbus based conjunctival openings in fellow eyes 2002;28:1758-62.

18. Wyse T, Meyer M, Ruderman JM, Krupin T, Talluto D. Am J Ophthalmol. Combined trabeculectomy and phacoemulsification: A one site versus two site approach 1998;125:334-39.

19. Das JC, Chaudhuri Z, Bhomaj S, Sharma P, Gupta R. Indian J Ophthalmol. Combined extracapsular cataract extraction with Ahmed glaucoma valve implantation in phacomorphic glaucoma. 2002;50:25-28.

20. al-Torbak A, Karcioglu ZA, Abboud E, Netland PA. Ophthalmic Surg Lasers. Phacomorphic glaucoma associated with choroidal melanoma 1998;29:510-13.

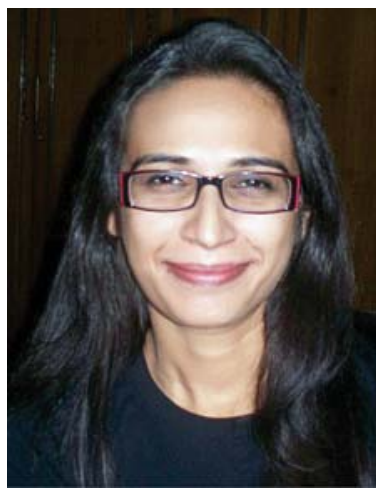

Shibal Bhartiya (shibalbhartiya@gmail.com)

"Those who live nobly, even if in their life they live obscurely, need not fear that they will have lived in vain. Something radiates from their lives, some light that shows the way to their friends, their neighbors, perhaps to long future ages.” 